\title{
Ropivacaine via trans-cricothyroid membrane injection inhibits the extubation response in patients undergoing surgery for maxillary and mandibular fractures
}

\author{
W. Gao ${ }^{1 *}$, J.H. Xi1 ${ }^{1 *}$, N.Y. Ju ${ }^{2}$ and G.X. Cui ${ }^{1}$ \\ ${ }^{1}$ Department of Anesthesiology, \\ The 2nd Affiliated Hospital of Harbin Medical University, Harbin, China \\ ${ }^{2}$ Department of Intensive Care, \\ The 3rd Affiliated Hospital of Harbin Medical University, Harbin, China \\ *These authors contributed equally to this study. \\ Corresponding author: G.X. Cui \\ E-mail: cuixiaoguangen@yeah.net
}

Genet. Mol. Res. 13 (1): 1635-1642 (2014)

Received June 18, 2013

Accepted October 29, 2013

Published March 12, 2014

DOI http://dx.doi.org/10.4238/2014.March.12.16

\begin{abstract}
Extubation response can lead to cardiovascular and respiratory complications. Here, we aimed to evaluate the effect of ropivacaine injected via the trans-cricothyroid membrane on the extubation response. This prospective, double-blind, randomized study included 70 patients classified as American Society of Anesthesiologists status I-II, who required general anesthesia with nasotracheal intubation for maxillary and mandibular fracture surgery; patients were divided into the ropivacaine $(20 \mathrm{mg})$ and dicaine $(20 \mathrm{mg})$ groups. Both groups were injected via the trans-cricothyroid membrane. Mean arterial pressure (MAP), heart rate (HR), and incidence and severity of cough were recorded during intubation and extubation. During intubation, there was no significant intergroup difference in MAP or HR and no occurrence of coughing $(\mathrm{P}>0.05)$. During extubation, MAP and HR
\end{abstract}


were significantly lower in the ropivacaine group than the dicaine group $(\mathrm{P}<0.05)$. The proportion of patients with no reports of cough was significantly higher in the ropivacaine group than in dicaine group $(\mathrm{P}<0.05)$. The number of patients with grade 1 or 2 cough was significantly higher in the dicaine group than that in the ropivacaine group $(\mathrm{P}<0.05)$. There was no significant intergroup difference in the rate of postoperative complications $(\mathrm{P}>0.05)$. These results suggest that the administration of ropivacaine via trans-cricothyroid membrane injection can effectively inhibit the extubation response.

Key words: Ropivacaine; Trans-cricothyroid membrane; Extubation

\section{INTRODUCTION}

Nasotracheal intubation is often a safer choice for the management of patients undergoing maxillary and/or mandibular fracture surgery (Wolfe, 1989; Ovassapian, 2001). The success of tracheal extubation during the patient's emergence from general anesthesia is critical. The extubation response can elicit undesirable cardiovascular and airway reflexes (Hartley and Vaughan, 1993; Kim and Bishop, 1998) and increases the rate of serious complications including laryngospasm, negative-pressure pulmonary edema and bleeding at the surgical site (Mendel et al., 1995; Nishina et al., 1995; Koga et al., 1998; Tagaito et al., 1998; Webster et al., 1999; Diachun et al., 2001; Soltani and Aghadavoudi, 2002). Furthermore, the open state of the mouth in maxillary and mandibular surgery may prevent successful re-intubation. Currently, lidocaine and dicaine are commonly administered by trans-cricothyroid membrane (TCM) injection to suppress the intubation response. However, the duration of action is short for both drugs (Bidwai et al., 1979). When an operation requires many hours, the extubation response cannot be inhibited effectively by dicaine or lidocaine.

Ropivacaine is a long-acting local anesthetic with intrinsic vasoconstrictive properties, and less cardio- and cerebral toxicity than bupivacaine (Groban et al., 2002; Stewart et al., 2003; Zink and Graf, 2008). Groeben et al. (2001) found that using ropivacaine in the airway to achieve topical anesthesia can significantly attenuate the severity of histamine-induced bronchospasm, but no previous study has examined the potential effect of ropivacaine on the extubation response. The aim of this prospective double-blinded study was to evaluate the ability of ropivacaine administered via TCM injection to inhibit the intubation and extubation responses.

\section{MATERIAL AND METHODS}

\section{Study design and patients}

This study was approved by the Ethics Committee of the Second Affiliated Hospital of Harbin Medical University and registered at the Chinese Clinical Trial Registry (ChiCTRTRC-12002343). Written informed consent was obtained from all patients. During the period from March to October 2012, 70 patients aged 20-60 years, classified as American Society of Anesthesiologists status I-II, who required general anesthesia with nasotracheal intubation for maxillary and mandibular fracture surgery, were enrolled. All patients were assigned 
randomly to either the ropivacaine or the dicaine group using a computer-generated number table $(\mathrm{N}=35)$. Patient assignments were recorded and sealed within sequentially numbered envelopes and were not revealed to the anesthesiologists. One anesthesiologist performed all TCM injections for both groups; any instance of cough and/or hemodynamic changes were recorded by a second anesthesiologist. The drugs for TCM injection were prepared by a third anesthesiologist, the one who had originally opened the envelope. Patients with concomitant cardiovascular disease, basal skull fracture, respiratory infection, nasal malformation, mental disorders, smoking history, gastroesophageal reflux, hiatal hernia, esophageal disease, and pharyngeal or epiglottic tumors were excluded.

\section{Anesthesia and treatment}

No pre-medication was administered. Blood pressure (BP), heart rate (HR), electrocardiography, and blood oxygen saturation $\left(\mathrm{SpO}_{2}\right)$ were continuously monitored, beginning 5 min after admission to the operating room. The radial artery was cannulated with a $22-\mathrm{g}$ catheter for real-time mean arterial pressure (MAP) monitoring. A single anesthesiologist induced topical anesthesia, performed the TCM injections and intubated all patients. The local anesthetics used prior to administering the TCM injections were provided in a blinded fashion by an independent assistant. Before the TCM injection, $0.1 \mathrm{mg} / \mathrm{kg}$ penehyclidine hydrochloride (an anticholinergic) and $0.03 \mathrm{mg} / \mathrm{kg}$ midazolam were injected intravenously to sedate the patient and reduce bodily secretions. In each case, the nasal airway mucosa and posterior pharynx were anesthetized topically with $7 \%$ lidocaine and $5 \mathrm{mg}$ ephedrine to induce vasoconstriction and reduce bleeding. After receiving a TCM injection of $20 \mathrm{mg} \mathrm{1 \%}$ ropivacaine or $20 \mathrm{mg} \mathrm{1 \%}$ dicaine, the patients were pre-oxygenated with $100 \%$ oxygen. The tube and nasal mucosa were smeared with lidocaine jelly to increase lubrication and reduce bleeding. Two minutes later, the patient was awakened for nasal tracheal intubation. If two attempts at intubation failed, the patient was excluded from the study. A successful nasotracheal intubation was confirmed with auscultation and end-tidal capnography. After intubation, the independent assistant injected propofol and rocuronium. Anesthesia was maintained with $10 \mu \mathrm{g} \cdot \mathrm{h}^{-1} \cdot \mathrm{kg}^{-1} \mathrm{remi}$ fentanil and $1.5-2 \%$ sevoflurane. The tidal volume was controlled at $7 \mathrm{~mL} / \mathrm{kg}$, the respiratory rate was set at 12 per min, and the expiration/inspiration ratio was set at 1:2. After returning to the ward, patients received an intravenous injection of $1 \mu \mathrm{g} / \mathrm{kg}$ fentanyl when they reported a visual analogue scale pain score $>4$.

\section{Evaluation}

The primary outcome measures for this study were the incidence and severity of cough during extubation. The secondary outcomes were the values of MAP and HR recorded at baseline (T1), upon tracheal intubation (T2), upon anesthetic withdrawal (T3), upon eye opening on verbal command (T4), upon tracheal extubation (T5), and 2 min after extubation (T6). A cough was recorded and graded by the second anesthesiologist according to the following scoring guidelines: 0 , no cough; 1 , slight cough, cough without obvious contraction of the abdomen; 2 , moderate cough, strong and sudden contraction of the abdomen lasting for less than $5 \mathrm{~s}$; and 3, severe cough, strong and sudden contraction of the abdomen sustained for more than $5 \mathrm{~s}$. 
Bleeding and infusion volumes were recorded. Five minutes after extubation, patients were transferred to the post-anesthesia care unit and evaluated with respect to potential adverse events including respiratory depression, hypoxemia and nausea/vomiting. Over the next $24 \mathrm{~h}$, any sign of nasal obstruction, nasal bleeding, oropharynx pain, and/or laryngeal edema was recorded.

\section{Statistical analysis}

Data were analyzed using the R2.10.0 software program. Values are reported as average and standard deviation. All data are reported as means (SD), number (proportion, $\%)$. Continuous variables were compared using two-factor repeated measures analysis. MAP and HR were compared at different time-points using the Student $t$-test. Categorical data were analyzed using the chi-square test or the Fisher exact test, when appropriate. $\mathrm{P}$ values $<0.05$ were considered to be statistically significant. A power calculation indicated that at least 70 patients would be needed to achieve a statistical power of more than $80 \%$.

\section{RESULTS}

Eight patients overall were excluded because two attempts at intubation had failed. There were no significant differences between groups with respect to demographic data, the duration of anesthesia and surgery, or bleeding and infusion volumes $(\mathrm{P}>0.05$; Table 1).

\begin{tabular}{|c|c|c|}
\hline & Group R $(\mathrm{N}=31)$ & Group D $(\mathrm{N}=31)$ \\
\hline $\operatorname{Sex}(M / F)$ & $16 / 15$ & $14 / 17$ \\
\hline Age (year) & $39.2 \pm 11.0$ & $41.4 \pm 12.5$ \\
\hline Weight (kg) & $67.2 \pm 8.7$ & $69.0 \pm 5.8$ \\
\hline Duration of surgery (min) & $80.0 \pm 18.8$ & $73.2 \pm 18.5$ \\
\hline Duration of anaesthesia (min) & $96.3 \pm 21.0$ & $92.2 \pm 21.8$ \\
\hline Volume of bleeding $(\mathrm{mL})$ & $133.9 \pm 28.4$ & $143.1 \pm 26.7$ \\
\hline Volume of infusion (mL) & $648.8 \pm 49.1$ & $665.2 \pm 50.6$ \\
\hline
\end{tabular}

$\mathrm{R}=$ ropivacaine group; $\mathrm{D}=$ dicaine group.

The incidence and grade of cough observed during intubation and extubation were compared between groups. No episode of cough was observed during intubation in either group. During extubation (time-points, T4 and T5), the proportion of patients without cough episodes was $91.9 \%(95 \% \mathrm{CI}=85.2-98.7 \%)$ in the ropivacaine group versus $46.8 \%$ $(95 \% \mathrm{CI}=34.4-59.2 \%)$ in the dicaine group $(\mathrm{P}<0.05)$. Twenty-six episodes of grade 1 cough $(41.9 \%)$ were observed in the dicaine group; 5 episodes $(8.1 \%)$ were observed in the ropivacaine group. Seven episodes of grade 2 cough (11.2\%) were observed in the dicaine group; none was observed in the ropivacaine group. The difference between groups was statistically significant $(\mathrm{P}<0.05$; Table 2$)$. In the dicaine group, the incidence of cough was $64.5 \%$ at $\mathrm{T} 4$ and $41.9 \%$ at $\mathrm{T} 5$; in comparison, the incidence of coughing in the ropivacaine group was 16.1 and $0 \%$ at T4 and T5, respectively $(\mathrm{P}<0.05$; Table 2$)$. 
Table 2. Comparison of incidence of coughing at $\mathrm{T} 4$ and $\mathrm{T} 5$ time-points.

\begin{tabular}{lccc}
\hline Grade of cough & $\mathrm{R}(\mathrm{N}=31)$ & $\mathrm{D}(\mathrm{N}=31)$ & $\mathrm{P}$ \\
\hline $\mathrm{T} 4$ & $26(83.9 \%, 70.9-96.9 \%)$ & $11(35.5 \%, 18.6-52.3 \%)$ & $<0.001$ \\
0 & 5 & 4 & \\
1 & 0 & 0 & $<0.001$ \\
2 & 0 & $18(58.1 \%, 40.7-75.4 \%)$ & \\
3 & & 3 & \\
$\mathrm{~T} 5$ & $31(100 \%, 100-100 \%)$ & 0 & \\
0 & 0 & & \\
1 & 0 & 0 &
\end{tabular}

MAP and HR values were significant lower in the ropivacaine group as compared to the dicaine group $(\mathrm{P}<0.05)$. MAP and $\mathrm{HR}$ at $\mathrm{T} 1, \mathrm{~T} 2$, and $\mathrm{T} 3$ were comparable; these values were similar in both groups ( $\mathrm{P}>0.05)$. MAP and HR values at T4, T5, and T6 were significantly lower in the ropivacaine $v s$ dicaine group; the ropivacaine group exhibited a more stable hemodynamic pattern than did the dicaine group at these time-points $(\mathrm{P}<0.05$; Table 3$)$. There was no significant difference between groups in terms of complications observed during the first 24 h postoperatively $(\mathrm{P}>0.05$; Table 4$)$.

Table 3. Comparison of haemodynamic profiles at different time-points $(\mathrm{N}=31$, means $\pm \mathrm{SD})$.

\begin{tabular}{|c|c|c|c|c|c|c|}
\hline & \multicolumn{3}{|c|}{ MAP (mmHg) } & \multicolumn{3}{|c|}{ HR (beats/min) } \\
\hline & Group R $(\mathrm{N}=31)$ & Group D $(\mathrm{N}=31)$ & $\mathrm{P}$ & Group $\mathrm{R}(\mathrm{N}=31)$ & Group D $(\mathrm{N}=31)$ & $\mathrm{P}$ \\
\hline $\mathrm{T} 1$ & $95.4 \pm 12.2$ & $93.8 \pm 7.3$ & 0.533 & $78.5 \pm 11.1$ & $78.9 \pm 10.7$ & 0.894 \\
\hline $\mathrm{T} 2$ & $94.3 \pm 15.3$ & $98.6 \pm 13.2$ & 0.241 & $79.2 \pm 10.2$ & $84.1 \pm 16.8$ & 0.163 \\
\hline $\mathrm{T} 3$ & $82.7 \pm 9.6$ & $83.5 \pm 7.7$ & 0.685 & $70.9 \pm 10.6$ & $71.4 \pm 10.0$ & 0.818 \\
\hline $\mathrm{T} 4$ & $90.9 \pm 10.9$ & $99.2 \pm 10.1$ & 0.025 & $79.5 \pm 10.3$ & $85.4 \pm 12.0$ & 0.042 \\
\hline $\mathrm{T} 5$ & $94.3 \pm 12.2$ & $101.6 \pm 10.9$ & 0.013 & $80.0 \pm 11.3$ & $87.5 \pm 12.9$ & 0.019 \\
\hline T6 & $90.8 \pm 12.2$ & $95.7 \pm 9.2$ & 0.049 & $76.0 \pm 9.9$ & $83.4 \pm 14.0$ & 0.016 \\
\hline
\end{tabular}

$\mathrm{MAP}=$ mean arterial pressure; $\mathrm{HR}=$ heart rate; $\mathrm{R}=$ ropivacaine; $\mathrm{D}=$ dicaine.

\begin{tabular}{llc}
\multicolumn{2}{l}{ Table 4. Comparison of the complications. } & \\
\hline Complications & Group & \\
\cline { 2 - 3 } & $\mathrm{R}$ & $\mathrm{D}$ \\
\hline Respiratory depression & 0 & 0 \\
Hypoxemia & 0 & 0 \\
Nausea/vomiting & 2 & 3 \\
Oropharynx pain & 5 & 6 \\
Nasal bleeding & 4 & 3 \\
Laryngeal edema & 0 & 0 \\
\hline
\end{tabular}

$\mathrm{R}=$ ropivacaine; $\mathrm{D}=$ dicaine.

\section{DISCUSSION}

The results of this investigation indicated that TCM injections of ropivacaine inhibited the extubation response more effectively than dicaine. Maxillofacial surgical procedures, 
especially maxillary and/or mandibular fractures, presented a unique set of problems for both the surgeon and the anesthesiologist. Nasotracheal intubation has proven to be a simple solution for maxillofacial surgical procedures. However, the period of emergence may be the most dangerous stage of maxillary and/or mandibular surgery. Anatomical and physiological changes contribute to a situation that can be more challenging than intubation for the anesthesiologist. A severe extubation response can significantly increase BP and HR (Aouad et al., 2009), potentially leading to postoperative hemorrhage, intracranial hypertension and/or intraocular hypertension (Leech et al., 1974; Saghaei et al., 2005; Irwin, 2006).

Currently, several methods are used to inhibit the extubation response during emergence, including deep extubation and the injection of lidocaine or opioids (Aouad et al., 2009). However, the advantages of these methods are offset by the potential for upper airway complications after extubation (Asai et al., 1998). Moreover, the intermaxillary wiring performed postoperatively may render subsequent re-intubation difficult. Leone et al. (2008) found that ropivacaine induced topical anesthesia and that the peak mean plasma concentration of inhalation is lower than the toxic threshold. In the present study, the author hypothesized that $1 \%$ ropivacaine via TCM injection could inhibit the extubation response more effectively than dicaine.

In clinical work and trials, the total number and grade of cough episodes during extubation indicates how smooth an extubation was (Fagan et al., 2000). In the present study, the authors observed and compared the cough, MAP and HR between groups at T1 and T2 to evaluate the effect of ropivacaine on the intubation response. The results showed that ropivacaine can be used for topical anesthesia and can inhibit the intubation response just as effectively as dicaine.

In this study, the grade and incidence of cough during emergence were significantly lower in the ropivacaine group than in the dicaine group; none of the patients in the ropivacaine group exhibited grade 2 coughing at the time of extubation. At T4, T5, and T6, MAP, and HR were significantly lower in the ropivacaine group as compared to the dicaine group. These results show that ropivacaine can significantly inhibit not only the intubation response, but also the extubation response. As a long-acting local anesthetic, ropivacaine can block impulse conduction in parasympathetic afferent or efferent nerve fibers and decrease the mechanical irritation of the airway caused by the presence of the endotracheal tube.

In this study, the extubation response was effectively inhibited by ropivacaine but not dicaine. Notably, while dicaine induces topical anesthesia lasting 40-60 min, ropivacaine induces topical anesthesia lasting over $2 \mathrm{~h}$. Compared with the effects of dicaine, those of ropivacaine last longer. In the context of topical anesthesia in the airway, ropivacaine is more effective in stopping neural conduction and inhibiting the airway reflex caused by contact with the endotracheal tube.

\section{LIMITATIONS}

The application of ropivacaine allows patients to tolerate the endotracheal tube well, minimizes hemodynamic changes and inhibits cough during emergence. However, the airway reflex is also a protective response: if the airway is blocked for a long time, the likelihood of postoperative complications increases. Further research is needed to investigate the time course of ropivacaine-induced topical anesthesia. 


\title{
CONCLUSIONS
}

In conclusion, the administration of ropivacaine via TCM is more effective than treatment with dicaine in terms of reducing the incidence and severity of cough as well as minimizing hemodynamic changes during the extubation period. Ropivacaine via TCM can be used to provide safe and comfortable extubation conditions for patients.

\section{Conflicts of interest}

There is no funding or research contract or conflict of interest.

\section{ACKNOWLEDGMENTS}

\author{
We would like to thank Professor Zhi Wen Li for extensive discussion of the manuscript.
}

\section{REFERENCES}

Aouad MT, Al-Alami AA, Nasr VG, Souki FG, et al. (2009). The effect of low-dose remifentanil on responses to the endotracheal tube during emergence from general anesthesia. Anesth. Analg. 108: 1157-1160.

Asai T, Koga K and Vaughan RS (1998). Respiratory complications associated with tracheal intubation and extubation. Br. J. Anaesth. 80: 767-775.

Bidwai AV, Bidwai VA, Rogers CR and Stanley TH (1979). Blood-pressure and pulse-rate responses to endotracheal extubation with and without prior injection of lidocaine. Anesthesiology 51: 171-173.

Diachun CA, Tunink BP and Brock-Utne JG (2001). Suppression of cough during emergence from general anesthesia: laryngotracheal lidocaine through a modified endotracheal tube. J. Clin. Anesth. 13: 447-451.

Fagan C, Frizelle HP, Laffey J, Hannon V, et al. (2000). The effects of intracuff lidocaine on endotracheal-tube-induced emergence phenomena after general anesthesia. Anesth. Analg. 91: 201-205.

Groban L, Deal DD, Vernon JC, James RL, et al. (2002). Does local anesthetic stereoselectivity or structure predict myocardial depression in anesthetized canines? Reg. Anesth. Pain Med. 27: 460-468.

Groeben H, Grosswendt T, Silvanus MT, Pavlakovic G, et al. (2001). Airway anesthesia alone does not explain attenuation of histamine-induced bronchospasm by local anesthetics: a comparison of lidocaine, ropivacaine, and dyclonine. Anesthesiology 94: 423-428.

Hartley M and Vaughan RS (1993). Problems associated with tracheal extubation. Br. J. Anaesth. 71: 561-568.

Irwin RS (2006). Complications of cough: ACCP evidence-based clinical practice guidelines. Chest 129: 54S-58S.

Kim ES and Bishop MJ (1998). Cough during emergence from isoflurane anesthesia. Anesth. Analg. 87: 1170-1174.

Koga K, Asai T, Vaughan RS and Latto IP (1998). Respiratory complications associated with tracheal extubation. Timing of tracheal extubation and use of the laryngeal mask during emergence from anaesthesia. Anaesthesia 53: 540-544.

Leech P, Barker J and Fitch W (1974). Proceedings: Changes in intracranial pressure and systemic arterial pressure during the termination of anaesthesia. Br. J. Anaesth. 46: 315-316.

Leone S, Di Cianni S, Casati A and Fanelli G (2008). Pharmacology, toxicology, and clinical use of new long acting local anesthetics, ropivacaine and levobupivacaine. Acta Biomed. 79: 92-105.

Mendel P, Fredman B and White PF (1995). Alfentanil suppresses coughing and agitation during emergence from isoflurane anesthesia. J. Clin. Anesth. 7: 114-118.

Nishina K, Mikawa K, Maekawa N and Obara H (1995). Fentanyl attenuates cardiovascular responses to tracheal extubation. Acta Anaesthesiol. Scand. 39: 85-89.

Ovassapian A (2001). The flexible bronchoscope. A tool for anesthesiologists. Clin. Chest Med. 22: 281-299.

Saghaei M, Reisinejad A and Soltani H (2005). Prophylactic versus therapeutic administration of intravenous lidocaine for suppression of post-extubation cough following cataract surgery: a randomized double blind placebo controlled clinical trial. Acta Anaesthesiol. Taiwan 43: 205-209.

Soltani HA and Aghadavoudi O (2002). The effect of different lidocaine application methods on postoperative cough and sore throat. J. Clin. Anesth. 14: 15-18. 
Stewart J, Kellett N and Castro D (2003). The central nervous system and cardiovascular effects of levobupivacaine and ropivacaine in healthy volunteers. Anesth. Analg. 97: 412-416.

Tagaito Y, Isono S and Nishino T (1998). Upper airway reflexes during a combination of propofol and fentanyl anesthesia. Anesthesiology 88: 1459-1466.

Webster AC, Morley-Forster PK, Janzen V, Watson J, et al. (1999). Anesthesia for intranasal surgery: a comparison between tracheal intubation and the flexible reinforced laryngeal mask airway. Anesth. Analg. 88: 421-425.

Wolfe SA (1989). Preoperative, intraoperative, and postoperative patient care in maxillofacial surgery. Clin. Plast. Surg. 16: 659-664.

Zink W and Graf BM (2008). The toxicity of local anesthetics: the place of ropivacaine and levobupivacaine. Curr. Opin. Anaesthesiol. 21: 645-650. 